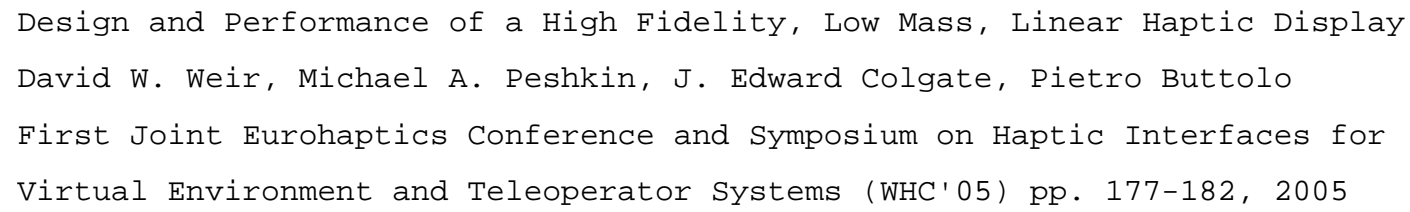

\title{
Design and Performance of a High Fidelity, Low Mass, Linear Haptic Display
}

\author{
David W. Weir ${ }^{*} \quad$ Michael A. Peshkin $^{*} \quad$ J. Edward Colgate ${ }^{*} \quad$ Pietro Buttolo $^{\dagger}$ \\ (*) Mechanical Engineering, Northwestern University, Evanston, IL USA \\ (†) Ford Motor Company, Ford Research Laboratories, Dearborn, MI USA \\ E-mail: \{d-weir, peshkin, colgate\}@northwestern.edu, pbuttolo@ford.com
}

\begin{abstract}
We built a high fidelity, low mass, linear haptic display, with a peak force output of 8.5 Newtons, continuous force output of 1.3 Newtons, range of motion of approximately 15 millimeters, sensing resolution of 0.5 microns, and a $-3 d B$ bandwidth of approximately $550 \mathrm{~Hz}$. By having low apparent mass of approximately 5 grams, we can realistically render linear switches, which themselves have a moving mass of only a few grams. This device utilizes a low inertia rotary motor, but over only a limited range of motion, allowing it to be driven without commutation. We constructed a linear current controlled amplifier to drive the system. Additionally, the motor windings allow us to use electrical damping to add physical damping to the system to improve its performance.
\end{abstract}

\section{Introduction}

Consider a typical development process for a console switch at an automobile company. This process usually starts with a jury of designers and engineers selecting a Best-In-Class (BIC) switch from internal and competitor's units. A supplier engineers a new switch using a sample of the BIC switch as a template, and a prototype switch is sent back to the automotive company. However, the prototype switch rarely feels like the selected BIC sample. The challenge is that currently there are few quantitative metrics to characterize switch-feel, and as such, no complete description can be given to suppliers and no method to verify their final product. In an ideal process, design requirements for switch-feel are quantitative and verifiable metrics based on psychophysical data. [1] To determine these metrics, as well as to verify the produced switch, an accurate system to characterize the physical properties of existing switches is needed. Creating such a system is the objective of this research.
Previous work included building a measurement device to capture the dynamics of human-switch interaction. [2,3] The measurement system is human actuated, allowing us to acquire real-life force, velocities, and accelerations. However, a playback device is needed to test the completeness of the measured data, referred to as the "haptic profile," and equally importantly, for use as a tool to specify and then further design the feel of switches. This paper describes the design and performance of the playback device or haptic display.

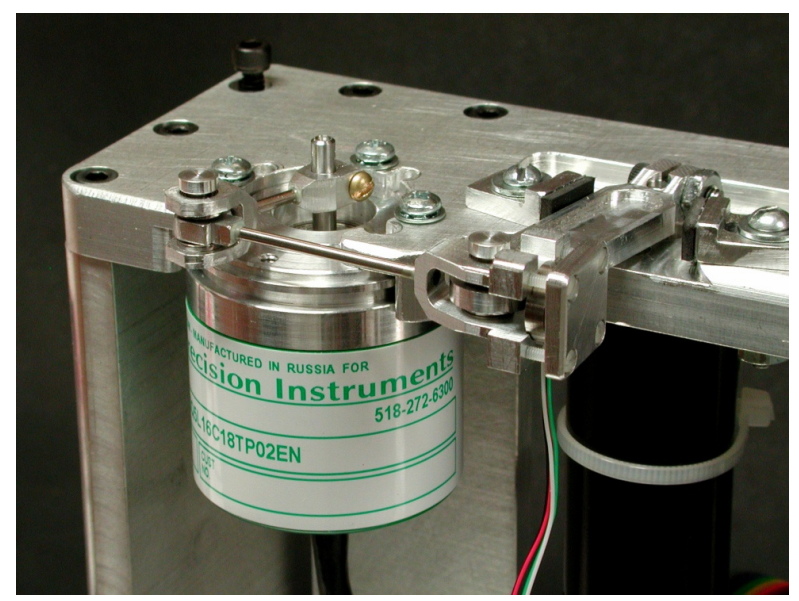

Figure 1. Isometric View of Haptic Display.

Similar research has been done using a mechanical system to measure and play back the haptic behavior of switches, however the measurement process was mechanically actuated, as opposed to human actuated, and the display device did not have the fidelity to fully render the resulting haptic experience. [4]

\section{Device Design}

A set of performance goals influenced many of the design decisions for this device. The goals were the force output, range of motion, endpoint position sensing resolution, measure user force, and maintain 
orientation of the user interface surface. The device had to have a peak user force output of at least 10 Newtons, a range of motion of at least one centimeter, a position sensing resolution close to 0.5 microns, and be able to sense the force exerted on the device by the user. In addition, it must maintain the orientation of the user interface surface, as opposed to allowing it to swivel or match the angle of the user's finger. At the same time, the design goals included reducing the apparent mass and internal friction of the system as much possible.

\subsection{Mechanical Design and Actuator Selection}

The design goal of making the apparent mass, the mass that the user "feels," as small as possible proved the most influential on the rest of the design decisions. Since our motivating task is simulating the dynamics of a linear switch, and typical linear switches have a moving mass of only a few grams, this is an ambitious goal. During the design process many actuators were proposed, such as using a linear motor, linear voice coil, rotary voice coil, brushed motor, or brushless motor. [5] Each system has benefits and detriments. The overall properties of the actuator types can be broadly separated into linear motion actuators and rotary motion actuators.

Linear actuators have a number of advantages. First, the motion is linear, like that of the push buttons we wish to simulate. Second, linear voice coils are non-commutated, simplifying the electronics design. The major disadvantage of the linear actuators is the force to mass ratio. In order to generate sufficient force output, the apparent, and in this case moving, mass of the actuator was significantly greater than in the rotary case, even when considering the addition of lever arms or linkages.

Conversely, the rotary actuator systems require an additional set of mechanical components to generate linear or near-linear motion. This adds mass and complexity to the system, but also allows the use of lever arms, which provide a large mechanical advantage to the user, reducing the apparent mass of the actuator. Standard rotary motors have a relatively large mass, but it is concentrated close to the axis of rotation, reducing the moment of inertia of the rotor. However, there is a tradeoff with rotary systems, because a longer lever arm reduces the effective output force of the motor, so a balance must be struck.

We found that the mechanical advantage gained by using a lever arm in a rotary configuration outweighed the loss of output force. A number of factors contributed to this, but the lever arm's mechanical advantage reducing the apparent mass of rotary actuators proved the dominant factor. Additionally, for a given torque output, there are a number of motor geometries available. Using a motor that employs a rotor with a high aspect ratio (length to width) was another important decision in further reducing the apparent mass and rotational inertia of the system. After searching available products, commercial rotary voice coils proved to be relatively more massive for the required torque output than standard rotary motors. To reduce cogging and friction, we selected a DC brushless rotary motor, specifically, the Maxon EC-22, 50 watt, model 167130 .

We chose to employ a four-bar linkage between the motor and encoder. This permitted the use of a rotary encoder; extremely high resolution encoders are readily available. The encoder serves a dual purpose; it both senses user position and also maintains the orientation of the interface surface, due to the linkage design. We chose a Gurley Precision Instruments rotary encoder, model $\mathrm{R} 137$, with a resolution of 230,400 counts per revolution. Surprisingly, the encoder added as much rotational inertia to the system as the motor.

Another rotary design advantage is using a shorter lever arm on the encoder than for the motor. By having a shorter lever arm, the encoder has more angular departure for a given user displacement, effectively increasing the resolution of the system. Also, since the encoder linkage does not experience any of the user's force, it can be made much lighter than the powered link connected to the motor. Since there is no external loading on the encoder half of the linkage, the encoder can sense the actual position of the user, independent of the deflection caused by the motor's or the user's torque on the powered link. Specifically, we designed a powered link with a 35 millimeter effective length, and a encoder sensing link with a 20 millimeter effective length. This causes a maximum lateral displacement, due to the arc shaped path, over the desired range of motion of only 0.36 millimeters. Also, since the sensing link is shorter than the powered link, the user interface surface does not stay exactly at the same orientation; there is a small angular deviation of 0.4 degrees. We conducted a very informal test of six people with two mockup devices; one that had two equal-length links of 35 millimeters and one that had the selected setup of one $35 \mathrm{~mm}$ link connected to a $20 \mathrm{~mm}$ link. The user interface force for both linkages moved over the same arc shaped path, however the unbalanced linkage also slightly rotated the user interface surface during actuation. In this quick test, it was not possible to distinguish the difference in user interface surface orientation (a 
deviation of 0.4 degrees over half the range of motion) between the two mockup devices.

A very small load cell is attached between the user interface surface and the powered link to measure the user force applied to the system.

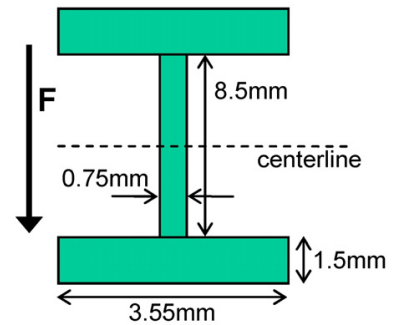

Figure 2. Cross Section of Powered Link.

The powered link is designed in such a way to minimize its mass, yet maximize its stiffness. Using an I beam shape with very thin webbing resulted in a total link apparent mass of less than 1 gram, while allowing an endpoint deflection of only 16 microns under a load of 25 Newtons. See Figure 2.

Originally, a magnesium alloy with a high strengthto-weight ratio (AZ31B or AZ80A) was selected to make the system components, however this proved logistically difficult, due to restrictions regarding the machining of (flammable) magnesium alloys in our machine shop. [6] Instead, a high strength aluminum alloy was selected as a replacement material, specifically 7075-T6. For this system, the difference in mass between a magnesium alloy and the 7075 aluminum alloy was between 10 and $15 \%$. This material has roughly twice the yield strength of more common aluminum alloys, such as 6061, although the modulus of elasticity is the same. Endpoint deflection under load was of concern, so the use of 7075 instead of 6061 did not change the overall mass or shape of the powered link, as the user force did not approach the yield strength of the material. Using 7075 aluminum alloy does provide a larger margin for damage in the case of instability of excessive user force, increasing durability.

\subsection{Electrical Design}

Since the mechanical design of the device utilizes rotary motion and the desired range of motion is small, this allowed us to use a single motor winding for the entire range of motion; that is, the motor is noncommutated. Non-commutation greatly simplifies the sensing and electrical design of the amplifier used to drive the motor. The motor selected has three windings, each offset by 120 degrees, and rotor has two poles; these attributes, combined with Maxon's clever winding geometry in the motor, make the torque output from a single winding sinusoidal, with a period of one complete revolution of the rotor. If the motor had more poles or windings, the period would decrease and a non-commutated system might not be possible.

The consequence is that the torque output of the motor is within $99 \%$ of the peak torque over a range of approximately 16.2 degrees, when the rotor angle is in the optimal angular position. See Figure 7 . Combining this with a powered link length of $35 \mathrm{~mm}$, produces a torque change over the desired 1 centimeter range of motion of only approximately $1.03 \%$, and the torque ripple over the entire $1.5 \mathrm{~cm}$ physical range of the device of only $2.3 \%$. Additionally, this torque ripple due to rotor angle can be easily compensated for in the controller software. Without commutation transients, the torque output of the device is very smooth and linearizable.

Also working in our favor is that we don't need to continuously simulate switches. Since switch actuation is a discrete action, the simulation needs only to be performed for a limited period of time. This allows us to overdrive the motor well beyond its continuous ratings. This is not an insignificant advantage; it allows us to achieve peak output forces 6.5 times greater than the continuous torque specification with our current electronics (the overdrive factor is presently limited by the voltage of our power supply). The ability to overdrive the motor can be used to create transient, high output features while rendering a virtual environment. The overdriving in fact must be transient to prevent thermal damage to the motor, due to high levels of power dissipated through ohmic heating in the motor winding.

We designed and built a high-current linear amplifier that acts as a controlled current source for the motor. This is implemented using a large, unregulated DC power supply, with the motor in series with an $\mathrm{H}$ bridge and array of power MOSFETs, acting as voltage controlled resistors. When simulating a switch, unlike typical applications of MOSFETs, they are acting like voltage controlled resistors, not in either the typical fully-on or fully-off mode, so the MOSFETs generate large quantities of heat, necessitating a parallel configuration to limit the individual power in each element. Even with four MOSFETs acting in parallel, they frequently dissipate more than 50 watts each during simulations.

The circuit is designed such that an analog command voltage is sent to the amplifier, which controls the motor current such that the voltage drop created across a small sensing resistor in series with the motor matches the command voltage. An analog, 
op-amp feedback loop around the sensing resistor controls the MOSFET resistance, and in doing so, controls the current through the motor. The analog feedback control is very fast, and we can precisely control the current through the motor, up to approximately 40 amperes, and therefore the force output of the display, since torque is linear with current for this motor. See Figure 3.

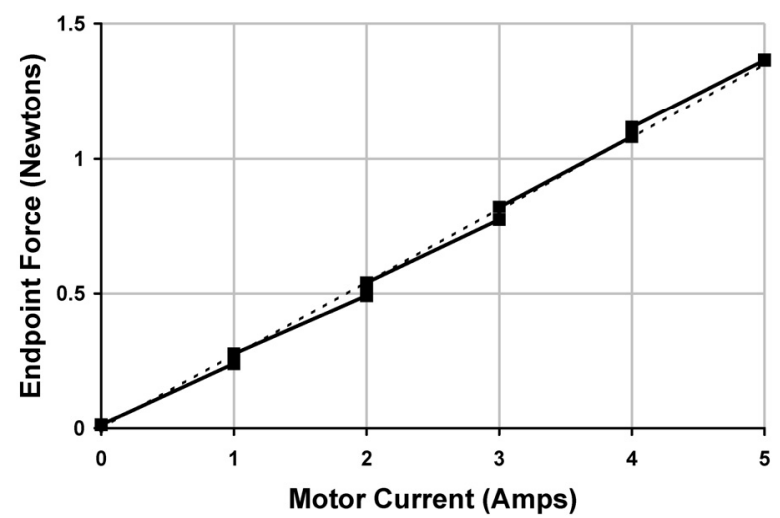

Figure 3. Motor Current to Endpoint Force Relation.

\section{Hardware Setup}

In order to accurately control the device and enable us to realistically render virtual environments, we employ the QNX real-time operating system using hardware interrupts to regulate the execution of our control software on a $266 \mathrm{MHz}$ Pentium II based computer. The control software is a multi-threaded application written in $\mathrm{C}$ for QNX. Data acquisition and signal I/O are handled by a Servo-To-Go Corp. interface board. The system servo rate can be set between 1 and $10 \mathrm{kHz}$; all performance data in this paper was collected at a servo rate $5 \mathrm{kHz}$. The servo sample timing error, while the system is recording data and controlling the haptic display, is symmetric and has a standard deviation of approximately 1.8 microseconds $(200 \mu \mathrm{sec} \pm 1.8)$. Performance data, such as the user force, endpoint position, motor torque, etc. was recorded at $5 \mathrm{kHz}$ as well.

\section{Device Performance}

The performance of the haptic display was broken down into two parts, first the electrical system was characterized, and then the mechanical (overall) performance of the display was measured. [7]

To measure the performance of our amplifier, a sinusoidal chirp command voltage, from 1 to 1000 Hertz, was generated, and the voltage across the current sensing resistor was measured. By comparing the two, we can determine the bandwidth of the electronics. Matlab provides an especially convenient method for doing this, by estimating the transfer function between input and output (tfe). The electrical bandwidth of the amplifier is flat past 1 kilohertz. See Figure 4.
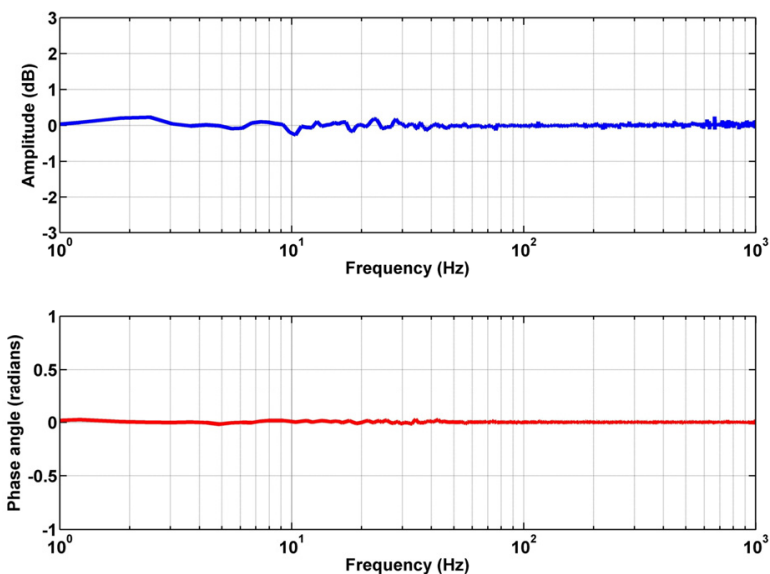

Figure 4. Amplifier Bandwidth (Commanded Current).

To determine the overall system response, a white noise command signal was used to drive the motor, while the command signal and output force were recorded. The haptic display has a $-3 \mathrm{~dB}$ bandwidth between command force and output force of approximately 550 Hertz. This measurement was performed with the user interface surface resting firmly against a heavy, stationary block of metal. The resulting data was again analyzed using Matlab's tfe algorithm. See Figure 5.
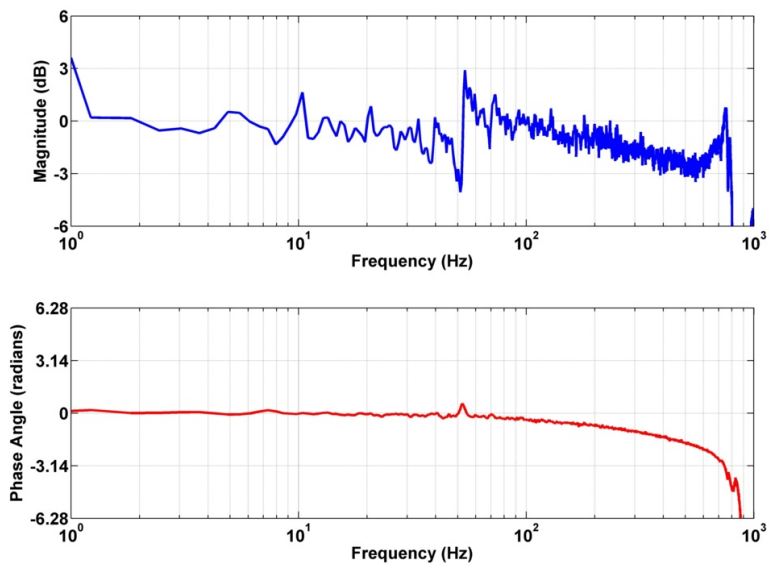

Figure 5. Commanded Force Bandwidth of Haptic Display.

For comparison, the performance of the display was also measured using the author's finger as the 
stationary surface resisting the display force. One should note that the magnitude scale is very different between Figure 5 and Figure 6 . Since the output performance of the haptic display is heavily damped when in contact with the user's finger and always below the commanded force bandwidth, we expect to be able to render haptic perceptions up to the bandwidth of the display. See Figure 6.
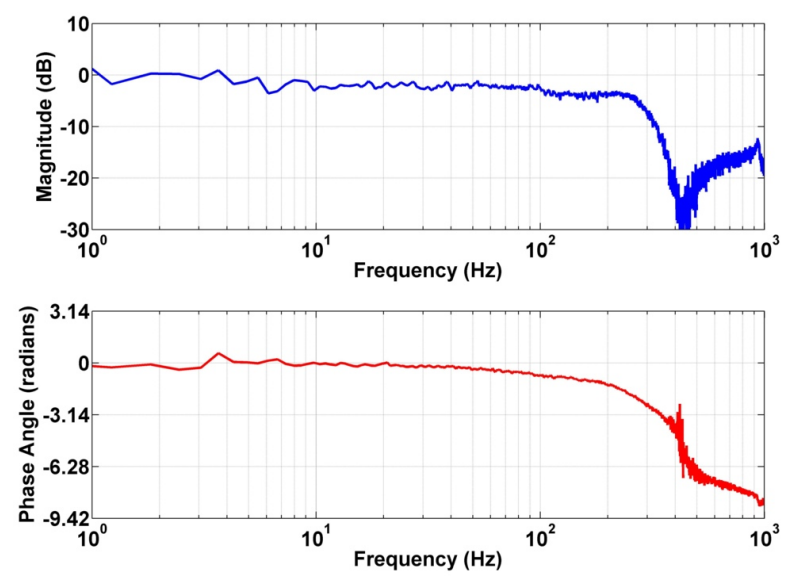

Figure 6. Display Bandwidth when Interacting with a Human Digit.

\section{Electrical Damping}

One future direction is to add damping to the system to increase its stability. As demonstrated in $[8,9]$, the performance of the display is limited by the mass and physical damping present in the system. One way of gauging the performance of a system is by using a factor known as the "Z-width" of the device. This is the ratio of the highest impedance interaction to the lowest impedance interaction that the system can stably render. That is, if in a virtual environment if free areas really feel free, and walls really feel solid/stiff.

One potential way to add damping to our haptic display is to add electrical damping to the amplifier system. The theory behind and a discussion of electrical damping is outlined in [10]. In our case, this may be accomplished using one of the unused motor windings to create damping. This is especially convenient, since the motor windings are in a "delta" circuit, as shown in Figure 7. To add damping, one of the windings could be shorted, partially crowbarring the motor. More sophisticated methods add damping only at high frequencies using frequency dependent filters.

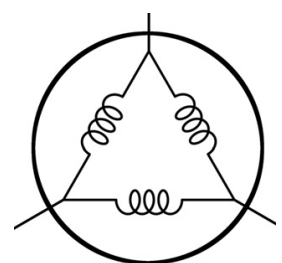

Figure 7. Motor Winding Diagram.

This use of real damping implemented electrically may stabilize the system and allow us to render stiffer, more realistic virtual features, such as virtual hard stops, detents, or walls.

Without electrical damping, the motor is normally driven by driving a current though one winding of the delta. This also creates a smaller current through the other two windings. In the case of simple electrical damping, one of the non-driven windings is shorted. As the rotor turns, the back emf induced in the winding creates a current, and a torque, that opposes the rotation of the motor, hence damping. Figure 8 illustrates the quasi-static relationship between motor torque and rotor angle in the normal case and also in the damped case. The damping added by the shorted winding is also shown on the plot. The damping is velocity dependent, Newton meters per radian per second, so the faster the rotor turns, the more resistance the damping provides. The torque and rotor angle relation indicates that when comparing a nondamped motor to a damped motor, the peak torque decreases by about $16 \%$, and the phase of the torque peak shifts by 30 degrees. See Figure 8 . The figure also illustrates that the damping is dependent on rotor angle, due to the phase dependent coupling between the rotor poles and the shorted winding.

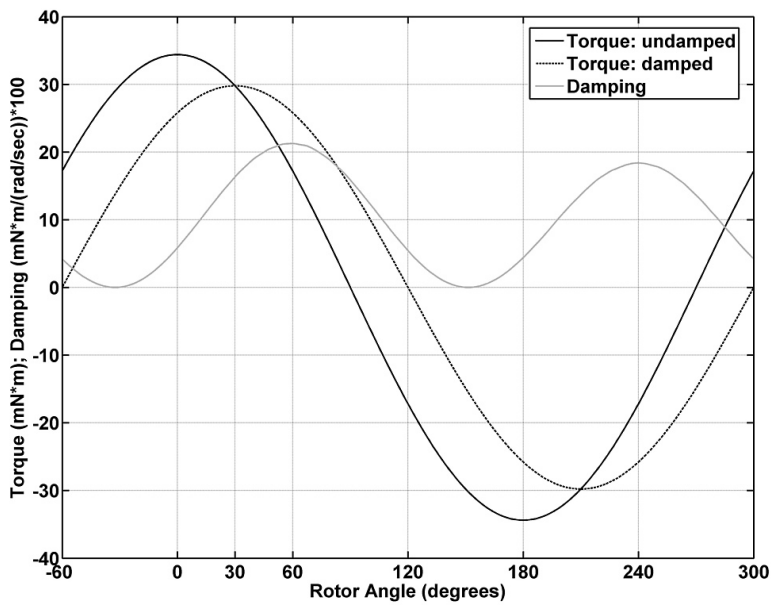

Figure 8. Measured Motor Torque-Rotor Angle Relation and Electrical Damping. 
At this time, these preliminary results indicate that electrical damping may be beneficial, however further investigation is needed to determine the quantity of physical damping added to the system using electrical damping. The change in system stability and performance is also a topic of further investigation.

\section{Conclusion}

We have rendered simple switch simulations using the haptic display and are developing more complex algorithms for playing back "haptic profiles" collected by our measurement system. The amplifier works as desired, and the system response seems appropriate for rendering switch interaction. Since the goal of this research is to use the haptic display as a design tool to specify and also explore switch-feel, further work needs to be done in developing techniques to transform the multidimensional data present in a haptic profile into something that can be rendered by the display. The development of control algorithms to allow us to make use of the full range of the haptic display will also be addressed in future work.

This work has been supported in part by the NSFIGERT program 'Dynamics of Complex Systems in Science and Engineering' (DGE-9987577).

\section{References}

[1] P. Buttolo, "Switch Measurement System", Ford Technical Report, 2001.

[2] D. Weir, M. Peshkin, J.E. Colgate, P. Buttolo, J. Rankin, M. Johnston, "The Haptic Profile: Capturing the Feel of Switches", Proceedings of the IEEE 12th International Symposium on Haptic Interfaces for Virtual Environment and Teleoperator Systems (Haptics 2004), Chicago, IL, March 27-28, 2004.

[3] D. Weir, Switch Characterization and the Haptic Profile, Masters Thesis, Northwestern University, Evanston, IL, 2003.

[4] K. MacLean, "The 'Haptic Camera:' A Technique for Characterizing and Playing Back Haptic Properties of Real Environments", Proceedings of the 5th Annual Symposium on Haptic Interfaces for Virtual Environments and Teleoperator Systems, ASME/IMECE, DSC-Vol. 58, Atlanta, GA, November 1996.

[5] P. Buttolo, B. Hannaford, "Pen-Based Force Display for Precision Manipulation in Virtual Environments", Proc. IEEE Virtual Reality Symp., 1995.
[6] J. G. Kaufman, Handbook of Materials Selection: Chapter 4 Aluminum Alloys; Chapter 8 Magnesium and its Alloys, M. Kutz Editor, John Wiley \& Sons, New York, 2002.

[7] V. Hayward, O.R. Astley, "Performance Measures for Haptic Interfaces", Robotics Research: The 7th Int. Symposium, Springer Verlag, 1996, pp. 195-207.

[8] J. M. Brown. A Theoretical and Experimental Investigation Into The Factors Affecting The Z-Width of a Haptic Display. M.S. Thesis, Northwestern University, March 1995.

[9] J. E. Colgate and J. M. Brown, "Factors Affecting the ZWidth of a Haptic Display." Proceedings of the IEEE International Conference on Robotics and Automation. San Diego, CA, Vol. 4, pp. 3205-10, 1994.

[10] J. S. Mehling. Electrical Damping as a Means for Increasing the Impedance Range of Haptic Displays. M.S. Thesis, Northwestern University, March 2004. 